\title{
EL CONOCIMIENTO PROFESIONAL DOCENTE \\ Y EL DISCURSO PEDAGÓGICO DE LA PRIMERA MITAD \\ DEL SIGLO XX. UNA CONTRASTACIÓN CON EL PERFIL, PARÁMETROS E INDICADORES DEL DESEMPEÑO DE LOS PROFESORES DE EDUCACIÓN BÁSICA EN MÉXICO
}

\author{
Héctor M. Jacobo García* \\ Universidad Pedagógica Nacional del Estado de Chihuahua
}

\section{RESUMEN}

En este artículo se explora el pensamiento pedagógico de la primera mitad del siglo xx a través del discurso de cinco voces educadoras de la época: G. Kerschensteiner, A.S. Neill, J. Dewey, E. Claparède y R. Ramírez. Establecidas las principales ideas pedagógicas de ese periodo, se compararon con la estructura holográmica y contenidos del perfil, parámetros e indicadores (PPI) del desempeño de los profesores y profesoras de educación básica (primaria) en México. Los resultados de la comparación son reveladores. La revisión hecha al discurso pedagógico identificó ideas clave que fueron reencontradas en el PPI. Algunas de ellas se refieren a lo que el educador debe saber acerca del desarrollo cognitivo y emocional de los alumnos, el aprendizaje, la enseñanza y cómo ha de ser su desarrollo profesional.

PALABRAS ClAVE: conocimiento profesional, holograma, formación de profesores, desarrollo profesional, pensamiento docente.

\section{THE PROFESSIONAL TEACHING KNOWLEDGE AND THE PEDAGOGICAL DISCOURSE OF THE FIRST HALF OF THE 20TH CENTURY. A COMPARISON WITH THE PROFILE, PARAMETERS AND INDICATORS OF THE PERFORMANCE OF BASIC EDUCATION TEACHERS IN MEXICO}

\section{Abstract}

This paper explores the pedagogical thinking from the first half of the 20th century through the distinguished discourse of five of the most remarkable educator voices in the time: G. Kerschensteiner, A.S. Neill, J. Dewey, E. Claparède, and R. Ramírez. Established the main ideas of the pedagogical thought of that period were compared with the hologramic structure and the contents of the profile, parameters and indicators of the performance of teachers of basic education (elementary) in Mexico. The results of the analysis are revealing. The revision made to the work of the aforementioned pedagogues, identified key ideas that were rediscovered in the PPI. Some of these ideas, for example, refer to what an educator needs to know about students' cognitive and emotional development, learning, teaching and how their professional development.

KEYwORDs: professional knowledge, hologramic, training teacher, professional development, thinking teacher. 


\section{INTRODUCCIÓN}

El estudio de la naturaleza del conocimiento profesional docente presenta avances importantes; sin embargo, las definiciones siguen dispersas, sin acuerdo resonante en la comunidad de profesores e investigadores y sin un impacto significativo en el trabajo pedagógico cotidiano (Clandinin \& Connely, 1987; Estebaranz, 2001; Tardif, 2004; Marrero, 1992, 1993, 2009).

¿Qué es lo que debe saber un educador? ¿Cuáles son los componentes de su saber y cómo se articulan? ¿Qué clase de conocimientos son los que identifican a la docencia como profesión? ¿En qué medida las diferentes piezas del discurso pedagógico de la primera mitad del siglo xx hacen concierto en el diseño del perfil de desempeño de los profesores de educación básica en México, así como la definición de los parámetros e indicadores (PPI) a los que se debe responder? Son preguntas que de un tiempo para acá me persiguen y de las que, sin tregua alguna e ininterrumpidamente, he sentido su acoso como una de las principales causas de mi malestar profesional.

A diferencia de Tardif (2004, pp. 135-137), al que le gusta menos el uso de las palabras saberes docentes y las cuestiona sin trascendencia aparente, conviene decir que en el presente estudio, dichos términos se refieren a todos los conocimientos, habilidades, actitudes y valores de los que el profesor dispone y con los que se moviliza para tener una conexión funcional con su entorno, reconocer los problemas profesionales originados en su lugar de trabajo y responder a ellos con un desempeño adecuado.

Por supuesto que las preguntas anteriores no son cosa de hoy, ni mucho menos inéditas. Tienen antecedentes muy remotos, pero con resonancias recientes y muy actuales. No obstante que la bibliografía sobre el tema sea vasta, se echa de menos en ella una definición y una caracterización consistentes. El diseño del PPI en el marco de la reforma educativa 2012-2013 en México parece que tiene algunas respuestas a las preguntas que cuestionan acerca de la naturaleza del conocimiento profesional docente.

Para referirse al tema, en este artículo se explora el pensamiento pedagógico a través del discurso de cinco voces autorizadas de la primera mitad del siglo xx: G. Kerschensteiner (Alemania). A.S. Neill (Inglaterra). J. Dewey (Estados Unidos de Norteamérica). E. Claparède (Suiza) y R. Ramírez (México).

Establecidas las principales ideas del pensamiento pedagógico de la época mencionada, se revisa la estructura y contenidos del perfil, parámetros e indicado-

*E-mail: hmjacobo@upnech.edu.mx. Es profesor normalista egresado de la Escuela Normal Superior de México y doctor en Psicología por la Universidad Autónoma de Madrid. Forma parte del Sistema Nacional de Investigadores, Nivel I (CONACYT), en México y de la Red Temática (CONACYT) de Investigación de Educación Rural y la Red Iberoamericana de Educación en Territorios Rurales. Actualmente se desempeña como profesor de contrato en la Universidad Pedagógica Nacional del Estado de Chihuahua, México. Inscrito en el marco de la complejidad social y cognitiva, tiene como línea de investigación el estudio de la naturaleza del conocimiento profesional, así como la formación y el desarrollo profesional docentes con énfasis en educadores de migrantes y de contextos adversos. 
res del desempeño de los profesores y profesoras de educación básica (primaria), se reflexiona con respecto al contexto en el que surge, se contrastan y se precisa lo que representa para la profesión docente.

\section{LOS SABERES DOCENTES EN EL CONCIERTO DE CINCO VOCES PEDAGÓGICAS DE LA PRIMERA MITAD DEL SIGLO XX}

En una revisión hecha a la obra de los pedagogos líneas arriba mencionados, se han recogido algunas ideas clave sobre lo que tiene que saber un educador acerca del desarrollo cognitivo y emocional, el aprendizaje, la enseñanza y cómo ha de ser su desarrollo profesional con tal de que cuente con las competencias para responder con acierto a las demandas pedagógicas de sus contextos de intervención.

\section{PRIMERA VOZ: SABER PRÁCTICO, TACTO PEDAGÓGICO Y TRABAJO EN COMUNIDAD}

G. Kerschensteiner (1854-1932), el supervisor de las escuelas de Múnich hace alrededor de un siglo, fue capaz de configurar una teoría acerca de lo que un profesor debe saber, se pronunció por la formación de un educador con mucho sentido práctico, que contara con el saber suficiente para hacer de la nueva generación ciudadanos útiles, capaces de establecer una relación funcional con su entorno, atendiendo la demanda social imperante.

Su fórmula pedagógica fue la Escuela del Trabajo. Era un tipo de escuela nueva caracterizada por el autogobierno que había sido pensada para tomar en cuenta y atender la individualidad infantil y en la que la acción pedagógica se ejercía de acuerdo a las necesidades de los niños. Ahí se estudiaban y exploraban las disposiciones innatas que la escuela habría de desarrollar (Kerschensteiner, 1912, p. 14.); se aprendía por experiencia y se combinaban el trabajo intelectual y el trabajo manual. Fue una escuela vinculada a la educación profesional, a la formación del carácter y a la educación cívica en la que se preparaba para la vida y la profesión futura; se aprendían la autorregulación de las propias acciones (Kerschensteiner, 1912, p. 19) y el altruismo.

Afirmó que el educador acierta de forma segura cuando coloca al amor hacia los demás en el centro de su trabajo pedagógico, si se conduce con tacto pedagógico, que es, por cierto, asunto de la intuición. Además, tuvo mucha confianza en el trabajo en comunidad de profesores para pensar juntos en el logro de la misión que los congregaba, siempre que se realizara con autonomía ${ }^{1}$, libre de jerarquías y vinculados

${ }^{1}$ Con respecto a la autonomía relativa de los centros escolares para que su personal docente en acuerdo con el director estructurara la enseñanza, Kerschensteiner argumentaba que «cuando se 
por una fraternal horizontalidad donde nadie es valorado por los títulos académicos que haya alcanzado, sino por lo que individualmente es (Kerschensteiner, 1934).

Kerschensteiner (1928) creía en el educador innato², por eso llegó a afirmar que la capacidad de ejercer influencia pedagógica era consecuencia del amor, y que por ello tal influencia dependía menos de los saberes adquiridos que de la sensibilidad psicológica innata y de la capacidad educadora con base en el tacto pedagógico.

En resumen, el ejercicio de la profesión docente para Kerschensteiner es cuestión de comprensión intuitiva, de amor a los niños, de sensibilidad psicológica, de tacto pedagógico y de hacer de la escuela una verdadera comunidad de profesores caracterizados por la fraternidad, en la cual los títulos académicos de cada uno de sus componentes no agregan valor a la persona, sino que todos en cuanto miembros de la comunidad escolar son estimados como seres individuales que están ahí no sólo para la cooperación cognitiva que requiere el ejercicio de la profesión docente, sino también para el apoyo mutuo y el soporte afectivo que cada profesor -como persona- debe encontrar en la comunidad escolar que constituyen ${ }^{3}$.

\section{SEGUNDA VOZ: PRAGMATISMO EN LA EDUCACIÓN DE LA MENTE PROFESIONAL DOCENTE Y OBJETOS DE REFLEXIÓN DIVERSIFICADOS}

John Dewey (1859-1952) fue un pedagogo de gran influencia en el periodo de referencia. Creó una teoría educativa con una red de conceptos clave con gran trascendencia e impacto para la comprensión de la vida social, del comportamiento humano y particularmente para construirle sentido a la acción educativa, pensándola de forma reflexiva y empoderando a la mente profesional para que recupere el

ordena todo, en conjunto y en sus detalles, por las autoridades superiores escolares y no es posible que un solo director, de acuerdo con el profesorado de la escuela, pueda llevar su iniciativa sobre métodos nuevos hallando instituciones escolares dentro y fuera de su respectivo centro, apenas si pueda esperarse que el director y el profesorado acierten a lograr una idea de la autonomía que a ellos mismos no les alcanza y que tampoco han conocido y experimentado en su propia formación» (1934, p. 97).

${ }^{2}$ Kerschensteiner (1928, p. 67) textualmente afirma: «Ciertamente es una felicidad para el género humano que la capacidad de ejercer influencia pedagógica, en tanto que brota como consecuencia del amor hacia el futuro portador de valores, dependa menos de los conocimientos pedagógicos y psicológicos adquiridos, que de la sensibilidad psicológica innata y de una capacidad educadora que se apoya en el tacto pedagógico».

${ }^{3}$ Kerschensteiner (1934, p. 98) cuando se refería al funcionamiento de las escuelas como verdaderas comunidades autónomas decía que no sólo era necesario que los alumnos aprendieran a vivir de forma más independiente a través de la práctica del autogobierno, sino también era necesario cumplir, como premisa fundamental, con «la existencia de un profesorado poseído de espíritu fraternal, en el que nadie se vea valorado por los títulos académicos que haya alcanzado, sino por su ser individual; donde son apreciados en una misma medida todos los que desempeńan una labor docente dentro de una escuela, donde todos se apoyen y auxilien mutuamente y colaboren unánimemente en los consejos que celebren; en una palabra, formando una verdadera comunidad, una colectividad fraternal animada por la idea de la misión que le está reservada». 
equilibrio de forma mayorante ${ }^{4}$ y proceda con certeza ante momentos de conflicto de inmanejable incertidumbre.

El hábito como síntesis dinámica entre el instinto/impulso y la cultura; la experiencia -lógica y psicológica- y la posibilidad de su reconstrucción, el pensamiento como proceso y como producto, la creencia en su parte estática y dinámica, el pensamiento reflexivo y los sentidos estratégicos de la acción; la comprensión evolutiva del desarrollo en el niño vinculado al programa escolar, la diversidad de teorías del aprendizaje y el poder desequilibrante y perturbador del conflicto, entendido como el tábano o acicate del pensamiento para generar un marco de perplejidad e incertidumbre que impele a la reorganización cognitiva ${ }^{6}$, configuran precisamente esa ecología de conceptos que requiere saber el profesor para pensar de otro modo la enseñanza e imaginarla creativamente en sus respuestas a la demanda de conocimiento del contexto educativo en el que ejerce la docencia como profesión.

Por supuesto que la apropiación significativa de estos conceptos contribuye a la educación del pensamiento del profesorado desde el punto de vista técnico; pero no lo es todo, tiene una demanda cognitiva mayor, implica que el profesor atribuya sentido a su trabajo, se sitúe en un marco de conflicto, identifique y ponga nombre al problema pedagógico que dicho conflicto genera; proceda a la búsqueda de la información necesaria para hacerla converger en una teoría que lo explique, formule después una hipótesis de trabajo y proceda a diseñar su intervención, la ponga a prueba, la evalúe, aprenda de ella y haga retroacción en caso de que los resultados no hayan sido los esperados y se inicie en un nuevo ciclo de aprendizaje en la profesión.

Conviene decir que, al vivir la experiencia psicológica de las acciones implicadas, el docente también se empodera cognitivamente para descentrarse del proceso

${ }^{4}$ Existen cuando menos dos referencias en las que J. Dewey se refiere al problema y al grado de dificultad para resolverlo, reestablecer el equilibrio y hacer posible el aprendizaje. En una de ellas afirma que «una gran parte del arte de la instrucción consiste en ampliar lo bastante la dificultad de los nuevos problemas para desafiar el pensamiento y que sea lo bastante pequeńa para que en medio de la confusión que naturalmente produzcan los nuevos elementos, haya luminosos puntos familiares de los que puedan brotar las sugestiones» (Dewey, 1927, p. 27). En la otra, refiriéndose a la posibilidad de elaborar juicios como el recurso que permite el restablecimiento del orden en la interacción, afirma: «Si la cuestión es íntegramente dudosa, si todo en ellas es tinieblas y oscuridad, entonces es un misterio absoluto, y tampoco en este caso tiene lugar el juicio. Pero si sugiere, aun cuando sólo sea vagamente, diferentes significados y posibles interpretaciones rivales, entonces hay en ella algún punto en cuestión, algo en juego. La duda toma forma de discusión, de controversia mental. Diferentes aspectos compiten por una conclusión que les favorezca» (Dewey, 1989, p. 112).

5 J. Dewey utilizó las palabras instinto e impulso indistintamente. En la edición en español de 1966 de su libro Naturaleza humana y conducta: introducción a la psicología social, publicada por primera vez en inglés en 1922, les atribuyó un significado prácticamente equivalente. En la nota al pie de la página 104, Dewey (1966) hace una aclaración sobre este tema.

6 Un análisis de materias acerca de las alusiones que J. Dewey hace de los términos conflicto, desequilibrio, perturbación, crisis, duda, incertidumbre, desconcierto, perplejidad para explicar cómo es posible el desarrollo y el aprendizaje puede leerse en las siguientes referencias bibliográficas: 1922, pp. 23, 46-47, 84, 180-182, 186 194, 200-201, 229, 254, etcétera; 1910, pp. 125, 127, etcétera; 1989, pp. 112, 115, 117, 121, 127, 225, etcétera. 
ejecutivo de la acción profesional, y proceder a revisarlo: hacer esto es un legado cognoscitivo de J. Dewey, y es, precisamente, el objeto de la reflexión práctica. Ayuda a reconocer la arquitectura del proceso, es decir, a identificar la estructura del guion o secuencia a la que responde el trabajo pedagógico realizado y permite, además, revisar la efectividad de sus componentes para cambiar lo necesario al reconocer los fallos en el proceso vivido.

Aprender haciendo y con base en problemas, experimentar la duda, la incertidumbre, la perplejidad y el conflicto, aprender junto con los demás, aprender de forma significativa y por descubrimiento son teorías a través de las cuales J. Dewey explicó el aprendizaje y planteó que proceder de acuerdo con ellas implica otro tanto de racionalidad técnica para sustentar la diversificación de la enseñanza.

Algo más que debe saber un educador es reflexionar sobre la función social de la educación. J. Dewey consideraba necesario educar la mente docente para que pensara su trabajo con responsabilidad social y se comprometiera con la formación de una persona capaz de establecer una relación armónica con su entorno.

\section{TERCERA VOZ: LA REFLEXIÓN TÉCNICA ANTES QUE EL CONOCIMIENTO PRÁCTICO PROFESIONAL DOCENTE}

E. Claparède (1873-1940) era un ferviente crítico del trabajo pedagógico hecho al tanteo. Se opuso a que se ejecutara sólo y a partir del buen sentido, al que descalificaba con la sentencia «el buen sentido, en efecto, afirma, pero no prueba» (1930, p. 39). No creía en la práctica docente sustentada sólo y a partir de la experiencia ${ }^{7}$, porque deviene en rutinas que sólo conducen a la reproducción mecánica de las prácticas profesionales.

Una enseñanza hecha sin sentido, ni reflexión alguna comprometida con la mejora, lo único que asegura es la costumbre mental orientada a la autopoiesis pedagógica. Cuando el educador se fía del automatismo, no ve las cosas como son, sino como ha tomado costumbre de verlas ${ }^{8}(1930$, p. 47). La rutina ciega a la razón,

7 Contra la experiencia no sistemática, E. Claparède afirmaba que era «... necesario, evidentemente, esforzarse por reducir al mínimo los servicios que se piden a la práctica, sobre todo cuando son seres humanos los que deben hacer el gasto. El maestro que aborda la práctica sin tener el menor conocimiento de la psicología, se halla naturalmente reducido a hacer tanteos, de los cuales son víctimas los alumnos; se ve obligado a hacer sus experimentos in anima vili, y algunas veces estos experimentos son muy largos y muy penosos para las generaciones de los alumnos que los sufren. Sin duda, la práctica puede, en cierta medida, suplir la deficiencia de los conocimientos teóricos; ipero, a precio de cuántos rodeos, de cuántos errores! Sin duda que a fuerza de construir puentes que se vienen al suelo, o máquinas que estallan, un técnico sin instrucción teórica acabará por terminar con buen fin estas obras, por encontrar empíricamente las fórmulas de construcción que él es incapaz de calcular. Pero, ¿quién querría los servicios de tal ingeniero?» (1930, pp. 53-54).

${ }_{8}^{8}$ Dice E. Claparède que «... el teórico, por oposición al práctico, es aquél que trata de establecer las causas exactas de los fenómenos, su coordinación, sus leyes, y que, gracias a esta elaboración de los datos que le suministra la observación de los hechos y el control experimental, se halla capaci- 
esteriliza al espíritu y se transforma en un obstáculo para el progreso científico porque no reconoce los descubrimientos ajenos ni deja que el educador los incorpore en su ejercicio profesional (1930, p. 49).

Eso se reflejaba también en la falta de conciencia técnica en el diseño curricular, dejando de lado las diferencias entre el sistema conceptual infantil y el adulto y la inconmensurabilidad existente entre dichos sistemas. Por eso Claparède sostenía que era muy importante traducir la experiencia del adulto en palabras, hechos y cosas que estuvieran en el repertorio lexical y de conocimientos de los nińos?.

Para escapar de la rutina e innovar la enseñanza y contar con una intervención pedagógica adecuada a los aprendices, Claparède hizo evidente la necesidad de sustentar la enseńanza en la ciencia. Era necesario, por lo tanto, que el educador fundamentara su trabajo pedagógico en la psicología, que conociera acerca del desarrollo de los estudiantes, sobre teorías del aprendizaje y otras ciencias de la educación para que efectivamente la marcha de la enseńanza se empatara a la marcha del desarrollo de los aprendices.

La diferencia técnica entre el curriculum escolar y la práctica docente, tanto como el déficit de saberes científicos en los profesores para fundamentar la enseñanza, la hizo notar en su libro Psicología del niño y pedagogía experimental (1930) y en su trascendente propuesta La escuela a la medida (1920) en la que ejemplificó magistralmente con la metáfora del sastre, el zapatero y el sombrerero. Argumentó que ninguno de los tres confeccionaba trajes, calzado o sombreros sin considerar la talla o las medidas físicas del destinatario. Fabricarlos a la medida del usuario era fundamental para evitar otros efectos. Hacer eso mismo, con la intervención pedagógica, diseñándola y ejecutándola, según fuera la inteligencia o grado de desarrollo alcanzado por los aprendices, lo convirtió en objeto de reflexión profesional y una de las tareas centrales del quehacer del educador.

tado para prever, inducir o concluir; porque ha aprendido a conocer el determinismo de los fenómenos. [Citando a Herbart, Claparede agrega que] "Herbart ha podido decir por eso [que] la práctica sólo produce la rutina, solamente la teoría nos enseńa a consultar la naturaleza" [Claparède continúa afirmando que] ... una larga rutina no basta, pues, dará aquél que la sufre una autoridad incontestable en materia pedagógica» (1930, p. 48).

${ }^{9}$ El espíritu del niño -explica E. Claparède- difiere además del maestro por la manera como organiza los conocimientos nuevos. Las experiencias de los adultos se hallan organizadas de una cierta manera muy adecuada, que tiene su ideal en las reglas de la lógica. Pero la lógica del niño no corresponde completamente a la del adulto; conviene, pues, traducir una a otra. Como esta traducción es difícil, no se hace habitualmente, y entonces, o bien se presentan al niño razonamientos de adulto que él no comprende o bien se despoja a la enseñanza de toda consideración lógica, se la reduce a un sencillo verbalismo, a un sencillo estudio de memoria, privándola así de lo que justamente podía constituir su valor (1930, p. 194). 


\section{¿QUÉ DEBE SABER Y SABER HACER EL EDUCADOR EN LA PROPUESTA DE CLAPARÈDE?}

Un educador debe saber psicología del niño, ha de saber cómo aprende y se desarrolla para fundamentar las decisiones en su trabajo pedagógico y evitar la enseñanza al tanteo. Debe contar con las habilidades para reflexionar de forma estratégica, buscando información con efectividad y haciéndola converger para fundamentar la intervención pedagógica.

Para Claparède, los educadores deben ser formados en la racionalidad científica y con las habilidades de pensamiento para reflexionar técnicamente sobre los problemas derivados del ejercicio de su profesión. Por esa razón, ante el conocimiento cotidiano y el buen sentido, opuso la teoría científica; y ante la práctica pura, la reflexión sustentada en el conocimiento de la psicología funcional. Deben ser competentes para diversificar la enseñanza: en eso también consiste La escuela a la medida. No debe dirigirse sólo a los nińos situados con capacidades intermedias, deben tener capacidades profesionales para generar distintas estrategias e intervenir con acierto a quienes tienen menos empoderamiento cognitivo y a los que sobresalen.

Algo más, en sus reflexiones éticas y filosóficas ${ }^{10}$ y el diseño de lo humano, Claparède profundizó menos. Como creía en la formación del hombre honrado y sano, independiente de espíritu, amigo del bien, de la verdad y la belleza, y con las virtudes físicas e intelectuales con respeto irrestricto de su personalidad (1930, p. 174), quería que los profesores aprendieran la reflexión en ese nivel con independencia de la enseńanza por considerar que ésta es independiente de la persona que se pretende formar, así como el modo de amasar la masa era independiente de la forma que habría de adquirir el pan.

10 «¿Cuál es el fin de la educación? -se preguntaba E. Claparède-. No se entiende la gente sobre este punto: hay tantas maneras de ver como personas. Cada cual, naturalmente, quisiera que la educación se hiciese en el sentido de sus preferencias sociales o políticas, filosóficas o religiosas; si se quisiese esperar a que todo el mundo se ponga de acuerdo sobre el fin que debe perseguirse, la ciencia de la educación no se fundaría nunca. Felizmente, el problema no es tan desesperado como parece. Así como la manera de amasar la masa es independiente de la forma que se dará al pastel, la manera de enseñar es, hasta cierto punto, independiente del fin último de la educación...». [En el párrafo siguiente, E. Claparède agrega] «... la instrucción -sobre todo la instrucción primaria y una buena parte de la secundaria- es un fin próximo casi completamente independientes del uso que se haga de ellas, y seguirán siendo las mismas, siendo cuales fueren los fines últimos del educador; la Aritmética, la Ortografía, la Física, la Geografía, etc. No hay una aritmética espiritualista y una aritmética materialista; como no hay una física burguesa y una física anarquista...». (1930, p. 174). 


\section{CUARTA VOZ: EDUCACIÓN CON JUSTICIA SOCIAL Y LA ESCUELA EN CONEXIÓN ORGÁNICA CON LA COMUNIDAD}

Rafael Ramírez (1885-1959), como ya se ha dicho en otro lugar (Jacobo, 2006), creía que los profesores deberían ser sujetos simpáticos, guiados por el sentido común ${ }^{11}$, que aplicaran el buen sentido en el ejercicio de su profesión. Está claro que no lo aceptó en los términos como lo hizo G. Kerschensteiner, quien se inclinaba por la imagen del educador práctico, pero tampoco lo rechazó con la fuerza y grado de convicción como lo hizo E. Claparède. Simplemente sabía que los profesores contaban con el sentido común en su desempeño profesional y que algunos de ellos decían que este tipo de conocimiento era bueno porque estimulaba su creatividad pedagógica ${ }^{12}$.

Comprometido con la escuela proletaria y en hacer de la educación el principal instrumento para el reparto de la justicia social, pensaba en la formación de un profesor insatisfecho con lo que sabe y hace y que cada día intenta saber más y hacer su trabajo mejor. Un profesional que sistemáticamente revisa su programa escolar para mejorarlo. Alguien que constantemente reflexiona sobre sus métodos de trabajo para remediar sus fallas o bien sustituirlos por otros más adecuados (Ramírez, 1935, p. 132), y sobre todo que se sabe inserto en una comunidad de vecinos en la que se representa y actúa como agente de desarrollo.

R. Ramírez tuvo un marcado interés por formar a los educadores con capacidad para fundamentar científicamente su trabajo pedagógico, de ahí que se planteara la formación de un profesor con la habilidad intelectual consolidada para reflexionar y fundamentar el trabajo pedagógico de acuerdo con la ciencia. Creía que al proceder así restaría inciertos al ejercicio de la profesión. Por esa razón en reiteradas ocasiones afirmó que el profesor ha de conocer cómo funciona la mente infantil, dado que el conocimiento psicológico es imprescindible para diseñar las estrategias de acción pedagógica más adecuadas para los sujetos que integraban el grupo esco-

11 «... pretendemos igualmente con estas lecciones -decía R. Ramírez, refiriéndose a su curso de técnica de la enseńanza- ayudar a los maestros rurales en servicio que no han tenido oportunidad de recibir un curso completo y ordenado sobre técnica de la enseñanza para que consigan que su actividad docente sea una cosa cada día más racional y más científica. El curso se apoyará, por un lado, en las nociones de psicología expuestas y explicadas... [más adelante afirma] ... se apoya, asimismo, sobre la experiencia y práctica de los estudiantes y maestros, pero, sobre todo, se inspira en el sentido común, ese sentido común que tan certera y sabiamente orienta la mayor parte de nuestros actos". (Ramírez, 1944, p. 144)

12 R. Ramírez cita los comentarios hechos por una profesora para validar el sentido común en el ejercicio de la profesión docente: «Maestro que quiere tener éxito enseñando a leer a los pequeños -comentaba la profesora-, despójese del fárrago de pedagogías que enturbian su pensar, y enseñe sencilla y naturalmente, siguiendo los dictados del buen sentido. Sobre todo use métodos que hagan agradable la enseńanza». "Yo trabajo según mi propio temperamento. Si todos los maestros trabajaran como yo, imitándome, entraríamos a una nueva rutina, que sería tan monótona y tan fastidiosa como la del silabeo. Dígales usted a los maestros, en sus lecciones de técnica de la enseñanza: ¡Por Dios, no sean ustedes ni maestros imitadores ni maestros rutinarios: sean maestros creadores sobre todo!» (Ramírez, 1944, pp. 309-312). 
lar. Tenía, además, una notable vocación por la individualización de la enseñanza. Identificado con E. Claparède y asumiendo la frase escuela a la medida, habló de la enseñanza a la medida como equivalente de aquella expresión, y utilizó la metáfora del overol ${ }^{13}$ con el mismo significado que E. Claparède atribuyó a la metáfora del sastre, el zapatero y el sombrerero.

Sin embargo, a diferencia de E. Claparède, y dado su compromiso de poner la educación al servicio de los pueblos socialmente retrasados - para decirlo como él-, pensaba que la escuela debería ser a la medida no sólo de las capacidades de cada nińo, sino también a la medida de las necesidades del desarrollo y potencialidades de la comunidad en que estaba inserta porque para R. Ramírez la escuela no era un apéndice de la comunidad, sino su corazón y la inteligencia misma. Estaba convencido de que era ahí, en la escuela, donde el profesor, los alumnos y los vecinos tenían que analizar colectivamente los problemas de su localidad; tomar conciencia de ellos; imaginar soluciones, planificar acciones y comprometerse con su ejecución.

Era una especie de trabajo social que sólo tenía efectos reales como educación comunitaria cuando todas las personas que integraban el pueblo se comprometían consciente y activamente en este proceso. Estaba convencido de que en la medida en que se analizaban los problemas de manera colectiva se estimulaba la toma de conciencia de los mismos y surgía la necesidad de resolverlos ${ }^{14}$. Una vez que aparecía la necesidad, lo demás llegaba por sí mismo, como, por ejemplo, la búsqueda y diseño de las estrategias, tanto como los procedimientos y recursos que se requerían para solucionarlos. En este caso el profesor se convierte en el consejero o guía del grupo que ha logrado problematizar su realidad y que sólo por ese hecho se empeña, desde ese momento, a jugar un papel más activo en el diseño del destino propio y el de su comunidad.

13 «Entendemos que este asunto de la individualización de la enseñanza -argumentaba R. Ramírez- [...], complica grandemente la tarea del profesor, pero entendemos también que si no se hacen esfuerzos para dar una enseñanza a la medida, los resultados de la educación serán mezquinos. La enseñanza dada al montón no resulta buena; se parece a esos overoles de pacota que a unas gentes les quedan zancones y apretados, mientras que a otras les quedan muy largos y demasiado amplios; el mejor overol es aquel que está hecho a la medida, a la medida en cuanto a tamaño y magnitud, así como en cuanto al estilo y método" (Ramírez, 1944, p. 333).

${ }^{14} \mathrm{R}$. Ramírez decía al respecto que había «dos modos o mejor dos métodos de desarrollar la acción social: el primer método es aquél en que el maestro se constituye él mismo en el iniciador y ejecutor de las obras de provecho comunal, quedando a los vecinos la simple tarea de ayudarlo en la realización de sus propósitos. Este método de trabajo no es educador, porque no capacita a los vecinos para que ellos mismos sean los que descubran las necesidades y problemas de su vida comunal y los resuelvan. El segundo método es aquél en que el vecindario mismo, no el maestro, se constituye en el iniciador y ejecutor de las obras de provecho comunal, reservándose el maestro el simple papel de guiar y aconsejar. Este método de trabajo social es realmente educador, porque enseńa y capacita a las comunidades para ser ellas mismas autoras de su propia transformación social» (Ramírez, 1944, p. 345). 


\section{QUINTA VOZ: REFLEXIÓN SOCIOCRÍTICA Y EDUCACIÓN SOCIOEMOCIONAL}

Alexander Sutherland Neill (1883-1973), en cambio, se interesó menos por el aprendizaje profesional en comunidad, pero hizo de Summerhill una comunidad democrática, con autogobierno. Promovió las asambleas como el espacio de interacción humana para el aprendizaje de la autorregulación en la que participaron todos los que formaban parte y hacían posible a Summerhill.

Como creador y director de la escuela de Summerhill, Neill dejó que los profesores trabajaran de manera individual y de acuerdo a sus costumbres metodológico-didácticas, y le importó poco que hicieran concierto pedagógico para aprender a reflexionar sobre la práctica de la enseñanza y generar conocimiento profesional. Lo muy importante para él era que los profesores no fueran moralistas ni inspiraran miedo en el niño (Neill, 1994, p. 153), y tuvieran, en cambio, un sentido del humor a toda prueba y el menor sentido de dignidad posible porque para el niño tener sentido del humor significa amistad, falta de respeto-confianza y ausencia de miedo.

Nunca estuvo de acuerdo con la fundamentación teórica de la enseñanza, cuando se refirió a ello afirmó que la mayor parte de sus escritos estaban sustentados en la observación de los niños, en la vida con ellos, y aunque reconoce que muchas de sus acciones fueron inspiradas por Freud, Homer Lane, Reich y otros, poco a poco fue abandonando la teoría, sobre todo en aquellos momentos en que la realidad le mostraba que no era válida (Neill, 1986, p. 85). Aunque no hizo de la intervención pedagógica su principal objeto de reflexión, prefirió mostrar cómo a través de la observación directa y tratando casos individuales se pueden generar saberes profesionales para tener mejores resultados. Creía en el efecto terapéutico del trabajo creador, por eso prefería que los niños hicieran más trabajo manual, más teatro y más danza (Neill, 1986, p. 49).

Propuso hacer de la libertad y el amor los principales pilares del aprendizaje de la autorregulación. Neill quería que los niños vivieran con alegría y felicidad, lo que se consigue cuando se actúa con bondad y se da sentido a la vida. Por eso él creía que no se debía intervenir en el desarrollo del niño porque se le perturbaba, lo mejor era dejarlo en plena libertad para que actuara conforme a sus deseos e intereses y lograr en cambio el desarrollo emocional que requería para que después el intelectual llegara por sí solo.

Lo recuperable en Neill para una caracterización de los saberes docentes es su capacidad para la observación sistemática del comportamiento espontáneo de los niños, y que es deseable para los educadores actuales. Otra habilidad intelectual importante a desarrollar en el educador es su capacidad para reflexionar con sentido crítico la función social de la educación, su conexión con la reproducción y el cambio social y educativo.

En resumen puede decirse que asomarse al pensamiento pedagógico a través de las voces e idearios de sus autores en el periodo aquí tratado permite esbozar la configuración y distinción más temprana de los principales rasgos del saber de los profesores. En el discurso pedagógico de los mismos aparece el componente racional científico (J. Dewey, E, Claparède y R. Ramírez), al creer que el trabajo 
pedagógico tendría que determinarse una vez que el educador sabe cómo aprenden y se desarrollan intelectualmente los alumnos; era muy importante que la enseńanza atendiera el desarrollo intelectual y que hecho esto, el desarrollo emocional vendría solo: por sí mismo; para otros, en cambio, el objeto central de la educación era el desarrollo emocional y que, al atender éste, el intelectual tendría cuidado de su propio curso. Así lo declaró A.S. Neill y en cierto modo también G. Kerschensteiner. Es por eso por lo que los primeros creían que el saber de los profesores era cosa de la ciencia; mientras que los otros afirmaban que era asunto del amor, de la intuición, de empatía y del tacto pedagógico. Para éstos, el profesor también es una persona, un ser cálido, sujeto de emociones, una máquina no trivial, impredecible; para aquéllos, un ser calculador, frío, sujeto de razón, una máquina trivial a la que, entrada una información, era predecible el resultado: la información de salida. Son concepciones heterogéneas que remiten a comportamientos docentes y de trabajo pedagógico diferentes. ¿¿Cuánto de ese saber acusa el desempeño actual de los educadores? ¿Qué contenidos del ideario pedagógico de las cinco voces anteriores configuran la intersubjetividad profesional consignada en el diseño del perfil, parámetros e indicadores de los profesores de educación primaria?

\section{EL CONCIERTO DE LAS CINCO VOCES PEDAGÓGICAS EN EL PERFIL, PARÁMETROS E INDICADORES PARA EL DESEMPEÑO DOCENTE DE LA EDUCACIÓN BÁSICA}

\section{ELementos DE CONTEXTO}

México es un país con la reforma educativa 2012-2013 en proceso y además cuestionada. Al implementarse trajo consigo controversias significativas, que dejaron contentos a unos, pero alentaron la inconformidad de muchos. Por supuesto que eso no fue casual, además de los inconfesables intereses de los grupos con poder de facto, hubo una diversidad de hechos que sofocaron e hicieron breve su vida productiva.

Desde el principio se cometió el error de homologar a la reforma educativa con la evaluación del desempeño docente, convirtiendo a ésta en el factor clave en el cual cifrar la calidad de la educación y la esperanza de generar las grandes y radicales reestructuraciones que requiere el viejo edificio del sistema educativo mexicano.

La evaluación fue simplificada y reducida a una herramienta puesta al servicio del ímprobo, por vertical y descontextualizado, esquema de vigilar y castigar para hacer evidentes los saberes docentes. Atribuirle carácter punitivo fue lo peor que pudo pasar: no sólo trajo consigo que el magisterio nacional se sintiera amenazado, también provocó su temprano desgaste como estrategia comprometida con la mejora de la enseñanza y los servicios educativos y evitó, incluso, la participación de los educadores para que se introdujeran como sujetos de conocimiento capaces de repensar y regular su quehacer, y generar en contexto y por sí mismos los saberes con los cuales dirigir su trabajo profesional con sentido y ser sujetos de autoevaluación. 
Los pensadores de la reforma educativa olvidaron que la máquina social es no trivial, sostenida por un sistema abierto, vivo y autoorganizado y que no admite la sustitución mecánica de los componentes que le dan identidad como se procedió con quienes no tuvieron resultados «idóneos» en la evaluación al desempeńo docente. Faltó humanizar la estrategia, proceder con empatía, escuchar todas las voces docentes, conocer las teorías de la mente y de los sentimientos de los educadores para proceder de forma inductiva y lograr que todos los procesos se implementaran con oportunidad y éxito.

Por otra parte, debe reconocerse que no obstante esos fallos y otros, la reforma educativa ha dejado aprendizajes importantes a todos sus actores. Ha puesto en evidencia, por ejemplo, que la implementación de arriba abajo, impuesta por la fuerza de los grupos de poder anidados en los partidos políticos y estructuralmente vinculados cual vasos comunicantes, sólo puede pararla una movilización política fuerte, como la de los profesores, que no sólo fue capaz de hermanar a un magisterio amenazado en su fuente de trabajo y organizado a la vez para la sobrevivencia profesional y el aseguramiento del sustento vital, sino que ha modelado en tiempos de elecciones públicas cómo utilizar el voto para decir que no a una política educativa insensata, neoliberal, configurada sólo y a partir de la convergencia de los intereses de los grandes oligarcas del país y más allá.

En ese caso debe reconocerse con objetividad que las respuestas del magisterio nacional han sido políticamente efectivas, pero técnica y académicamente débiles. No emergen de movimientos consolidados de renovación pedagógica, protagonizados por educadores con propuestas consistentes de educación alternativa, por lo que a falta de éstas, la reforma resultó ser técnicamente incontestable; no existe respuesta profesional posible que reoriente o revierta el verticalismo del que deriva. Sin duda, la lucha de los maestros ha sido una reacción colectiva emocionada, fuerte, pero lamentablemente con un proyecto de educación inconsistente y poco legible para una gran parte de las mentes que participan en el movimiento de protesta.

Como contrasentido al verticalismo con el que se han implementado los procesos de reforma, se ha promovido en los centros escolares, en cambio, la autonomía de gestión y el trabajo colegiado a través de la integración de las comunidades para el aprendizaje de la profesión docente y el desarrollo profesional. Los propósitos son múltiples, entre ellos conviene destacar haber logrado que las comunidades contribuyan a la construcción de un conocimiento profesional que ayude, entre otros temas, a situar la enseñanza y la formación, que permita comprender el papel que juegan los contextos en la constitución social y psicológica de los sujetos escolares, tanto como en sus aprendizajes. Las sesiones mensuales de consejo técnico escolar (CTE) que tienen lugar en los centros escolares son reveladoras al respecto.

Un aspecto relevante de la cuestionada reforma educativa, quizá el más importante para la reflexión que se tiene en curso, fue el diseño del PPI del desempeño docente, con base en el cual la Coordinación Nacional del Servicio Profesional Docente de la SEP, con la aprobación técnica del Instituto Nacional Para la Evaluación de la Educación (INEE), evalúa el desempeño de los profesores de educación básica y media superior, concesionándola al Centro Nacional de Evaluación (CENEVAL). 
El PPI es un instrumento necesario, que permite establecer la configuración de la autorreferencia y cultura e identidad profesionales porque tipifica y formaliza los saberes y prácticas que identifican al ejercicio de la profesión docente en los diferentes niveles de la educación básica, y media superior.

La precisión del mismo permitió establecer la versión oficial de los límites entre lo que es y no es la docencia como profesión de Estado e hizo visible su carácter holográmico al suponer que el conjunto total de la cultura de la profesión docente está en cada educador, al mismo tiempo que éste se socializa en la profesión, adquiere identidad docente y forma parte de la totalidad. En ese sentido, es válido decir que el PPI compendia todos los saberes de la profesión que han de orientar y regular el ejercicio de las intervenciones educativas de los docentes.

Si bien los PPI se diseñaron mediante una consulta nacional a profesores del nivel de educación correspondiente, y en cierto modo lo dicho allí ha sido tomado como teorías implícitas (Marrero, 2009) o teorías en uso a través de las cuales el educador actúa como tal, también es cierto que ha sido revelador el carácter intersubjetivo de las mismas y en el que hacen concierto las cinco voces pedagógicas descritas en la primera parte de este capítulo.

En ese sentido, en las cinco dimensiones ${ }^{15}$ que integran el PPI se compendia no sólo el saber docente como conocimiento práctico profesional y teorías en uso, sino que incluye, además, una serie de conceptos clave identificados con el discurso de los cinco pedagogos antes mencionados.

\section{LAS DIMENSIONES DEL PPI Y LAS VOCES PEDAGÓGICAS EN CONCIERTO}

Sabiendo que no hay nada nuevo bajo el sol, lo que este ensayo pretende es reivindicar al diseño del PPI como una contribución trascendente para la docencia como profesión, no obstante las objeciones magisteriales a la mencionada reforma educativa y que a la fecha (septiembre de 2018), la crónica del anuncio de su muerte haya empezado a escribirse.

Conviene advertir que la tipificación del saber y quehacer de la docencia como profesión no es inédita, su existencia data de mucho antes de la promulgación de las bases jurídicas de la reforma educativa 2012-2013, pero que tuvo el atino

${ }^{15}$ Las dimensiones integradoras del perfil de desempeño docente son:

Dimensión 1. Un docente que conoce a sus alumnos, sabe cómo aprenden y lo que deben aprender. Dimensión 2. Un docente que organiza y evalúa el trabajo educativo y realiza una intervención didáctica pertinente.

Dimensión 3. Un docente que se reconoce como profesional que mejora continuamente para apoyar a los alumnos en su aprendizaje.

Dimensión 4. Un docente que asume las responsabilidades legales y éticas inherentes a su profesión para el bienestar de los alumnos.

Dimensión 5. Un docente que participa en el funcionamiento eficaz de la escuela y fomenta su vínculo con la comunidad para asegurar que todos los alumnos concluyan con éxito su escolaridad. 
de formalizarlos en el PPI a través de la consulta hecha a los maestros de México, recuperando su conocimiento disciplinar, normativo, contextual, las teorías en uso y buenas prácticas.

Entre los discursos de las cinco voces pedagógicas aquí citadas, es posible identificar algunos conceptos asociados a las distintas dimensiones que integran el PPI. Movilizados en la primera mitad del siglo pasado, actualmente esos conceptos mantienen suficiente consistencia y configuran diversos campos semánticos de identidad profesional. Se trata de una red de saberes teóricos y prácticos extendida por toda la cartografía multidimensional del mencionado instrumento. Eso prueba que el PPI cuenta con una parte invariante de saberes y prácticas delegada por la cultura pedagógica cuya relación funcional con los contextos educativos le ha permitido resistir a su extinción.

Existen varios argumentos para creerlo; entre otros los siguientes, al menos uno de ellos por cada dimensión del perfil:

\section{Argumento 1}

En la dimensión 1 se tipifica el conocimiento que han de tener los profesores con respecto a sus alumnos. Deben conocer acerca de cómo aprenden los alumnos y lo que deben aprender. Esta necesidad del saber profesional no es nueva, es insoslayable y fundante, además, del quehacer del educador. Se trata de un saber inscrito en el perfil de desempeño que coincide con el interés de los pedagogos para que los profesores explicaran el aprendizaje con base en teorías psicológicas científicas y superaran el modo convencional inscrito en el conocimiento de la experiencia básica cotidiana. Dewey, Claparède y Ramírez mantuvieron explicaciones cognitivas del aprendizaje, muy cercanas a lo que se conoce con el nombre de teorías del aprendizaje significativo (Ausubel, Novak \& Hanesian, 1983), por conflicto (Piaget, 1978) o por interacción social espontánea asociada al aprendizaje cooperativo (Johnson, Johnson \& Holubec, 1999).

Los tres pedagogos también asumieron una teoría interaccionista del desarrollo en la que se concibe que el empoderamiento cognitivo del individuo está en proceso y en constante evolución que remite a una sucesión de síntesis dinámicas transitorias resultantes de la interacción de la naturaleza del individuo con el entorno natural y sociocultural en el que vive. Ellos mismos consideraron que esta forma de entender el desarrollo era una condición cognoscitiva de los educadores para que lograran un trabajo pedagógico con acierto, porque sólo cuando se conoce el curso y ritmo del desarrollo intelectual de los niños es posible empatar la marcha de la enseñanza, adecuándola a él.

Kerschensteiner y Neill partieron de una teoría innatista. Ambos creían que las personas ya habían nacido con una estructura interna -espiritual o mental- y que el desarrollo consistía sólo en encontrar el entorno sociocultural adecuado para que sus potencialidades innatas tuvieran mundo posible para desenvolverse. Por eso ambos pedagogos reivindicaron la libertad; el primero con su Escuela del Trabajo y el segundo con la Escuela de Summerhill. Son proyectos educativos en los que 
se reivindica el conocimiento práctico del profesor, en los que se atiende la estructura profunda de los niños, reivindicando el amor, la comprensión, la empatía, el tacto pedagógico y la autonomía como base sobre la cual generar un ambiente de aprendizaje en plena libertad. En ambos se cree que el aprendizaje es posible siempre que haya una identificación entre la estructura interna del sujeto y las que aporta el entorno sociocultural, por eso la importancia de la diversificación de los escenarios y las oportunidades de interacción. No obstante, el sistema de creencias de los pedagogos que sustenta a ambas propuestas, no existe un solo indicador del desempeño docente inscrito en algún parámetro del perfil que efectivamente conceda tantos grados de libertad a la intervención educativa.

\section{Argumento 2}

La dimensión 2 supone a un docente que sabe organizar y evaluar su trabajo educativo y que realiza una intervención didáctica pertinente, por lo que organiza su intervención docente para lograr el aprendizaje de sus alumnos, identificando sus características y diseñando estrategias de intervención docente para atender las necesidades educativas. Éste es un saber docente técnicamente considerado en los discursos pedagógicos de la primera mitad del siglo pasado. E. Claparède lo advirtió en su propuesta La escuela a la medida y R. Ramírez con la metáfora del overolo del sombrerero. El primero decía que la enseñanza debe ser como los trajes que confeccionan los sastres, todos diferentes, adaptados a la individualidad con tal de hacerlos a la medida. Esa misma idea retomó Rafael Ramírez para decir que los overoles y los sombreros también deben considerar la talla del que los porta.

Algo más, cabe reconocer que las voces pedagógicas con ideas interaccionistas acerca del desarrollo movilizan sugerencias prácticas sobre la planificación de la enseñanza, creen conveniente que los educadores tengan control cognoscitivo de la condición psicológica para aprender y que se conozca la demanda cognitiva de un contenido de aprendizaje para poder apropiárselo y que de acuerdo con ello diseñen la intervención pedagógica y organicen los grupos escolares para vivir la experiencia. Por esta razón Ramírez (1944, pp. 31-32) sugirió a los maestros elaborar tres programas diferentes y tres métodos según fueran las capacidades de los niños: baja, media o alta. También dijo que se podía intervenir al grupo de alumnos con un solo método, siempre que al final del trabajo pedagógico se dedicara tiempo extra para la atención individualizada de quienes más lo requerían.

\section{Argumento 3}

La dimensión 3 establece que un docente debe reconocerse como profesional que mejora continuamente para apoyar a los alumnos en sus aprendizajes, inscrito en el parámetro 3.1, y coincidente con el indicador 3.1.2, en el que se espera que un profesor idóneo sea capaz de utilizar diferentes referentes teóricos para analizar su práctica y mejorarla en su rediseño. Los tres pedagogos interaccionistas 
(Dewey, Claparède y Ramírez) ya lo tenían previsto, querían precisamente que los profesores fueran capaces de pensar su trabajo pedagógico y conceptuar su práctica docente con base en el saber académico; aunque Ramírez mostró simpatía con el conocimiento práctico docente, hizo causa común con los otros dos en su oposición a la repetición mecánica del trabajo pedagógico sin reflexión y sin fundamento científico. Así lo refleja, por ejemplo, J. Dewey en su concepción de pensamiento reflexivo magistralmente expuesto en su multicitado libro Cómo pensamos y E. Claparède en la crudeza crítica con la que se refería a la enseñanza de los educadores hecha al tanteo, como lo consigna en su trascendente libro Psicología del niño $y$ pedagogía experimental.

Las otras dos voces pedagógicas -Kerschensteiner y Neill-creyeron menos en la racionalidad técnica de la enseñanza. El primero se opuso a la enseñanza intervencionista porque estaba convencido de que el profesor no educa y que la mejor educación es aquélla que lo hace superfluo y ocurre con la libertad suficiente para que viviera la experiencia psicológica de acuerdo al llamado de sus propias inclinaciones o de sus gestalten sin génesis. Cada quien actúa de manera espontánea y cuenta con opciones para autoelegir las actividades que mejor se acomoden a sus intereses.

Muy próximo a esas concepciones, Neill refleja cierto desdén por la enseñanza. No promovió la innovación didáctica y dejó a los profesores que trabajaran de acuerdo a la tradición pedagógica en la que estaban instalados. Igual que con los niños, también fue permisivo con el personal docente de Summerhill. Los dejó en libertad y, quizá por ello, no logró romper con las prácticas pedagógicas tradicionales en el contexto de las aulas, mientras que en el contexto de la escuela, hacía de la libertad y el amor los principales métodos de enseñanza. Neill como educador, en cambio, sí promovía que sus lecciones fueran interesantes, creativas y que echaran a volar la imaginación de los niños, pero esto no era un comportamiento docente común en Summerhill. Los demás profesores, en el inmovilismo pedagógico, hacían autopoiesis pedagógica en el ámbito de los métodos de enseñanza.

\section{Argumento 4}

La cuarta dimensión se refiere a las capacidades del docente para asumir las responsabilidades legales y éticas propias de la profesión para el bienestar de sus alumnos. Con respecto al conocimiento de la normativa institucional las cinco voces pedagógicas exponen el reconocimiento a la misma como regulador de las prácticas institucionales; sin embargo, Neill, aunque la observó por obligación, también la despreció. Sabía que era necesario responder a las regulaciones estatales, pero la dinámica interior escolar no la organizó con ese propósito. Es cierto que no desconoció su responsabilidad, pero tampoco perturbó el desarrollo de los niños, obligando su asistencia a clases con tal de que contaran con la preparación previa para dar la mejor respuesta a los exámenes de la supervisión educativa del reino. Cada niño tomaba la decisión por sí mismo de acudir a clases y prepararse para los exámenes. Afirma Neill que cuando esto ocurría, los niños 
obtenían muy buenos resultados y todo era posible porque ya estaban curados del daño que les había hecho la sociedad hipócrita en la que habían vivido durante los años previos a Summerhill.

Algo más que conviene destacar es que tanto en la Escuela del Trabajo como en la de Summerhill se promovía el respeto, la colaboración y la más sana convivencia, similar a la que se expresa en los indicadores del parámetro 4.2 del PPI, en el que se demanda del profesor establecer un ambiente favorable para la sana convivencia y la inclusión educativa en su práctica docente.

\section{Argumento 5}

La quinta dimensión del PPI espera que un docente participe activamente en el funcionamiento eficaz de la escuela en la que está adscrito y que fomente el vínculo con la comunidad para asegurar que todos los alumnos terminen con éxito su escolaridad. En esta dimensión el vínculo con la comunidad es relevante y el discurso pedagógico del periodo mencionado le concede un lugar importante. En la revisión hecha, se encontró que todos ellos hacen referencia al vínculo que los educadores de un centro escolar han de establecer con la comunidad. Kerschensteiner (1912, p. 119), por ejemplo, afirmaba que muchos de los problemas didácticos experimentados por el profesor tenían relación estrecha con el contexto comunitario en el que estaba inserta la escuela porque dependían de las circunstancias de tipo económico, espacial y temporal. Además de tener activo el vínculo con la comunidad de vecinos, quería que el personal hiciera de su escuela una comunidad. La pensó integrada por personas tramando relaciones horizontales, libre de jerarquías y promoviendo el diálogo profesional más prolífico con tal de que los niños recibieran la mejor atención educativa. En esta perspectiva, todo vínculo comunitario hacia afuera o hacia adentro de la escuela, se establecía en relación con los niños con el interés de favorecer el nivel de logro cognoscitivo de cada uno de ellos.

R. Ramírez, en cambio, se vinculó con la comunidad no tanto para conocer el entorno de los alumnos y proceder a hacer las adecuaciones curriculares y de enseñanza con tal de imprimir mayor calidad a los procesos de enseñanza y aprendizaje; lo hizo para llevar la escuela a la comunidad y que funcionara como la inteligencia misma en la cual se discutirían los problemas que constriñen el bienestar de la gente, en este caso el vínculo con la comunidad pretende fines de justicia social y no psicopedagógicos.

\section{CONCLUSIONES}

1. En esta contrastación entre los componentes del PPI y las ideas que expresan cada una de las cinco voces pedagógicas se ha hecho visible que gran parte de los saberes que agrupa de forma organizada el PPI han sido dichos en el discurso pedagógico generado durante la primera mitad del siglo Xx. 
2. Independientemente del contexto en el que se ha diseñado, el PPI supone un importante avance en la definición y organización de los saberes que cada uno de los prácticos de la profesión docente debe portar consigo.

3. Que efectivamente la formalización de los saberes y quehaceres en el PPI representa el holograma de la profesión docente, dando consistencia y facilitando la identidad profesional y la cultura que le es propia y que para ser internalizada se requiere vivir la experiencia psicológica de los procesos de socialización que suelen ocurrir en los lugares de trabajo docente.

Recibido: 19-04-2019. Aceptado: 24-05-2019 


\section{REFERENCIAS}

Ausubel, D., Novak, J. y Hanesian, H. (1983). Psicología educativa. Un punto de vista cognositivo. México: Ed. Trillas.

Clandinin, J. y Connelly, M. (1987). «Teachers personal knowledge: what counts as "personal” in studies of the personal». Journal Curriculum Studies, 19 (6), 487-500.

Claparède, E. (1930). Psicología del niño y pedagogía experimental. Madrid: Francisco Beltrán, editor.

Claparède, E. (1920). La escuela a la medida. Madrid: Ediciones de la Lectura, S.A.

Johnson, D.W., Johnson, R.T. y Holubec, E.J. (1999). Aprendizaje cooperativo en el Aula. Buenos Aires: Paidós Educador.

Dewey, J. (1967). El niño y el programa escolar. Mi credo pedagógico. Argentina: Losada.

Dewey, J. (1966). Naturaleza humana y conducta, introducción a la psicología social. México: Fondo de Cultura Económica, $4 .^{a}$ ed.

Dewey, J. (1933). How we think. A restatement of the relation of reflective thinking to the educative process. Lexington, MA: D.C. Heath and Company.

Dewey, J. (1927). Obras de Dewey IV. Los fines, las materias y los métodos de la educación. Madrid: Ediciones de la Lectura-Impresiones Ciudad Lineal.

Dewey, J, (1922). Human nature and conduct. New York: Henry Holt and Company.

Dewey, J. (1989). Cómo pensamos. Nueva exposición entre el pensamiento reflexivo y proceso educativo. Barcelona: Paidós.

Estebaranz, A. (2001). "La naturaleza del conocimiento del profesor», en Memoria del Simposium Internacional en Formación de Profesores. Culiacán, Sinaloa, México: Secretaría de Educación Pública y Cultura del Gobierno del Estado de Sinaloa.

Estebaranz, A. y Sánchez, V. (eds.) (1992). Pensamiento de profesores y desarrollo profesional I. Conocimiento y teorías implícitas. Sevilla, España: Secretariado de Publicaciones de la Universidad de Sevilla.

Jacobo-García, H.M. (2006). Teoría Educativa. Lo que todo profesional de la educación tiene qué saber. México: UPN.

Kerschensteiner, G. (1912). Concepto de la escuela del trabajo. Madrid: Ediciones de la Lectura-Impresiones Ciudad Lineal.

Kerschensteiner, G. (1928). El alma del educador y el problema de la formación del maestro. Barcelona: Editorial Labor.

Kerschensteiner, G. (1934). La educación civica. Barcelona: Editorial Labor.

Marrero, J.E. (2009). EL pensamiento re-encontrado. Barcelona: Octaedro.

Marrero, J.E. (1993). «Las teorías implícitas del profesorado: vínculo entre la cultura y la práctica de la enseñanza», en M.J. Rodrigo, A. Rodríguez y J. Marrero (eds.). Las teorías implícitas una aproximación al conocimiento cotidiano. Madrid: Visor.

Marrero, J.E. (1992). «Las teorías implícitas del profesorado: un puente entre la cultura y la práctica de la enseñanza", en A. Estebaranz y V. Sánchez (eds.). Pensamiento de profesores y desarrollo profesional. Volumen I. Conocimiento y teorías implícitas, (pp. 9-22). Sevilla, España: Universidad de Sevilla, Secretariado de Publicaciones. 
NeILl, A.S. (1986). Summerhill. Un punto de vista radical sobre la educación de los niños. Madrid: Fondo de Cultura Económica.

Neill, A.S. (1994). El nuevo Summerhill. México: Fondo de Cultura Económica.

Piaget, J. (1978). La equilibración de las estructuras cognitivas. México: Siglo XXI, Editores.

RAMíREZ, R. (1935). «El interés mundial por la educación de los grupos socialmente retrasados», en Obras completas de Rafael Ramirez, tomo v, Veracruz, México: Dirección General de Educación Popular.

Ramírez, R. (1944). «Técnica de la enseñanza», en Obras completas de Rafael Ramírez, tomo III, Veracruz, México: Dirección General de Educación Popular.

SEP (2018). Perfil, Parámetros e indicadores para los docentes de educación primaria. México: SEP. Disponible en: http://servicioprofesionaldocente.sep.gob.mx/2017/ba/PPI/PPI_DOC_TECNICO_DOCENTES_080118.pdf.

TARDif, M. (2004). Los saberes del docente y su desarrollo profesional. Madrid: Narcea. 
